\title{
Morphometric evaluation of the delayed cerebral arteries response to acetazolamide test in patients with chronic carotid artery stenosis using computed tomography angiography
}

\author{
A. Szarmach ${ }^{1}$, P.J. Winklewski ${ }^{1}$, G. Halena ${ }^{1}$, M. Kaszubowski² , J. Dzierżanowski ${ }^{1}$, M. Piskunowicz ${ }^{1}$, \\ E. Szurowska ${ }^{1}$, A.F. Frydrychowski ${ }^{1}$ \\ ${ }^{1}$ Medical University of Gdansk, Poland \\ ${ }^{2}$ Department of Economic Sciences, Faculty of Management and Economics, Gdansk University of Technology, Gdansk, Poland \\ [Received: 11 March 2016; Accepted: 28 May 2016]
}

Background: The evidence accumulates that the response to acetazolamide test is delayed on the ipsilateral side to stenosis. However, the effect of acetazolamide beyond 30 min after acetazolamide administration remains unknown. The aim of this study was to assess the diameters of anterior cerebral arteries (ACAs), middle cerebral arteries (MCAs) and posterior cerebral arteries (PCAs) before and $60 \mathrm{~min}$ after the acetazolamide test.

Materials and methods: Seventeen patients with carotid artery stenosis $\geq 90 \%$ on the ipsilateral side and $\leq 50 \%$ on the contralateral side were enrolled into the study. Diagnosis was based on ultrasonography examination and was confirmed using digital subtractive angiography. In all patients, two computed tomography angiography examinations were carried out; the first was performed before the acetazolamide administration, while the second one was carried out 60 min after injections.

Results: In response to the acetazolamide test: PCA diameter diminished in both ipsi- and contra-lateral side to stenosis (from 1.31 to $1.24 \mathrm{~mm}$ and from 1.23 to $1.15 \mathrm{~mm}$, respectively), ACA and MCA decreased in the contralateral side to the stenosis (from 1.33 to $1.26 \mathrm{~mm}$ and from 2.75 to $2.66 \mathrm{~mm}$, respectively), ACA and MCA increased in the ipsilateral side to the stenosis (from 1.29 to $1.46 \mathrm{~mm}$ and from 2.77 to $2.96 \mathrm{~mm}$, respectively). All changes were statistically significant. Conclusions: There were significant differences in reactivity to acetazolamide challenge between the internal carotid artery (ICA) and vertebrobasilar circulation in patients suffering from chronic carotid artery stenosis. Within the ICA territory, $A C A$ and MCA responses vary in the affected and not affected side. (Folia Morphol 2017; 76, 1: 10-14)

Key words: cerebral arteries, computed tomography angiography, acetazolamide test, cerebral regional reactivity

\section{INTRODUCTION}

Cerebral blood flow (CBF) and its distribution are highly sensitive to changes in the partial pressure of arterial carbon dioxide $\left(\mathrm{CO}_{2}\right)$. This physiological response, termed cerebral $\mathrm{CO}_{2}$ reactivity, is a vital homeostatic function that helps to regulate and maintain central pH [2]. Physiological studies suggest regional differences in $\mathrm{CO}_{2}$ regulation of blood

Address for correspondence: P.J. Winklewski, MD, PhD, Institute of Human Physiology, Medical University of Gdansk, ul. Tuwima 15 , 80-210 Gdańsk, Poland, e-mail: pawelwinklewski@wp.pl 
flow between the internal carotid artery (ICA) and vertebrobasilar circulation. Lower $\mathrm{CO}_{2}$ reactivity in the vertebrobasilar system may be beneficial for preserving blood flow to the medulla oblongata to maintain vital systemic functions, while higher $\mathrm{CO}_{2}$ reactivity in the ICA system may imply a larger tolerance for varied blood flow in the cerebral cortex $[16,17]$.

In clinical practice, the acetazolamide test is often used to estimate autoregulatory reserve in subjects with ICA stenosis. Acetazolamide is a reversible inhibitor of the enzyme carbonic anhydrase, and although the exact mechanism is unknown, the vasodilatory effect on the vasculature of the brain is ascribed to carbonic acidosis [19]. Cerebral perfusion of the brain tissue is measured before and after a vasodilatory challenge. The evidence accumulates that the response to the acetazolamide test is delayed on the ipsilateral side to stenosis [10, 13]. Hartkamp et al. [10] measured reactivity till $30 \mathrm{~min}$ after acetazolamide infusion while $\mathrm{Mu}$ rakami et al. [13] completed the experiments after $20 \mathrm{~min}$. Therefore the delayed response beyond this timeframe remains unknown. We have previously shown that in patients population with chronic carotid artery stenosis (CAS) within the frontal grey matter there are no differences in $\mathrm{CBF}$ response to the acetazolamide test in ipsi- and contra-lateral sides to the ICA stenosis [7]. We speculated that the diameter of the ipsilateral anterior cerebral artery (ACA) and middle cerebral artery (MCA) may need to dilate more than the contralateral ACA and MCA to accommodate the increased CBF.

The aim of this study was to assess the diameters of arteries originating from the circle of Willis (CoW), namely: ACA, MCA and posterior cerebral artery (PCA), before and 60 min after acetazolamide test. Based on the above presented considerations, we hypothesised that in response to acetazolamide, the PCAs diameters diminish regardless of the side due to blood steal effect. We also expected ACA and MCA diameters to decrease in the contralateral side to stenosis, and increase in the ipsilateral.

\section{MATERIALS AND METHODS}

\section{Patients}

Seventeen patients with CAS $\geq 90 \%$ on the ipsilateral side and, $\leq 50 \%$ on the contralateral side were enrolled into the study. Diagnosis was based on ultrasonography examination and was confirmed using digital subtractive angiography (DSA). In all patients, the duration of CAS was longer than 5 years. The experimental protocol and the study were approved by the ethical committee of the Medical University of Gdansk. All volunteers gave written informed consent to participate in the study. Patients with stage II-V renal insufficiency were excluded.

\section{Angiography}

Confirmatory DSA was performed prior to carotid artery stenting. Patients were considered for this study when the internal CAS exceeded $90 \%$, and the stenosis on the contralateral side was less than $50 \%$ (using NASCET method). Selective angiography of the target vessel was then performed to reveal the size of the carotid lesion, degree of stenosis, morphology of the ICA and its contribution to the CoW. Only patients with complete CoW and normal vertebral arteries were included into this study.

\section{Computed tomography angiography}

In all patients, two computed tomography angiography (CTA) examinations were carried out; the first was performed before acetazolamide administration, while the second was carried out $60 \mathrm{~min}$ after injections of $1.0 \mathrm{~g}$ Diamox i.v. (Mercury Pharmaceuticals, London, UK). All of the CTA were performed with a 64-MDct Light Speed VCT XT scanner (GE Healthcare Technologies, Chicago, Illinois, US).

The protocol for CoW CTA included 0.625-mm slice thickness cuts with following parameters of the examinations: rotation time $-0.4 \mathrm{~s}$, pitch $-0.984 \mathrm{~mm}$, the interval $-0.625 \mathrm{~mm}$, tube voltage $-120 \mathrm{kVp}$, tube current $-335 \mathrm{mAs}$, matrix of $512 \times 512$, display field of view $-250 \mathrm{~mm}$. Then, $60 \mathrm{~mL}$ of non-ionic contrast media (Iomeron 250, Bracco Imaging Deutschland, Constance, Germany) was injected at $4 \mathrm{~mL} / \mathrm{s}$ with standard-concentration $(300 \mathrm{mgl} / \mathrm{mL})$ through a large-bore intravenous access in the antecubital vein, followed by a chaser bolus of $30 \mathrm{~mL}$ injected at the same rate.

\section{Imaging protocol}

Reconstructions of the raw data were performed using the separate workstation (Advantage 4.4; GE Healthcare, Chicago, Illinois, US).

The diameters of MCA, ACA and PCA were analysed using maximum intensity projections perpendicularly to the long axis of the each artery in these some points twice: before and after acetazolamide administration. 
To minimise measurement errors we adjust professional software (GE ADW ver. 4.4) initially dedicated to the evaluation of the coronary arteries. The diameters of each vessel (M1, A1, P1) were automatically measured by two radiologist. The final result was the average value of the measurements for each artery.

\section{Statistical analysis}

Data parameters were presented as mean \pm standard deviation. Normality assumption was verified with W Shapiro-Wilk test. The differences between values of ACA, MCA and PCA diameters before and after acetazolamide test were examined by paired test or Wilcoxon matched pairs test, if necessary. The level of significance was set at $\alpha=0.05$. All raw data were analysed using statistical software Statistica 10 (StatSoft, Tulsa, OK, US).

\section{RESULTS}

Complete CoW, including open both posterior communicating arteries, in all patients participating in the study were confirmed in DSA examination. In response to acetazolamide test:

- PCA diameter diminished in both ipsi- and contralateral side to stenosis;

- ACA and MCA diameters decreased in the contralateral side to the stenosis;

- ACA and MCA diameters increased in the ipsilateral side to the stenosis.

Posterior carotid artery response to acetazolamide challenge was quite homogeneous, out of 34 analysed arteries, the decline in diameter was observed in 24 (70.6\%) PCAs, in 10 (29.4\%) PCAs there was no change, while there were no arteries with increased diameter. In all patients at least diameter of one PCA was diminished.

On the contralateral side to the stenosis the ACA diameter was narrowed by $5.3 \%$, while MCA by $3.3 \% 60$ min after acetazolamide administration. The ipsilateral ACA diameter was augmented by $13.2 \%$, while MCA by $6.9 \% 60 \mathrm{~min}$ after the acetazolamide challenge.

Detailed results are provided in Table 1. Figure 1 shows axial scans before and after acetazolamide test. Figure 2 provides CTA 3-dimensional reconstruction in coronal plane with the left ICA narrowed by approximately $80 \%$ by atherosclerotic plaques.

\section{DISCUSSION}

There are two main findings of the study: 1) there are significant differences in reactivity to acetazola-
Table 1. Changes in anterior carotid artery (ACA), middle carotid artery (MCA) and posterior carotid artery (PCA) diameters before and after acetazolamide challenge in the ipsi- and contra-lateral side

\begin{tabular}{llcccc}
\hline & $\begin{array}{l}\text { Hemi- } \\
\text { sphere }\end{array}$ & $\begin{array}{c}\text { Mean } \\
\text { diameter }\end{array}$ & $\begin{array}{c}\text { Stand- } \\
\text { ard de- } \\
\text { viation }\end{array}$ & t/z & $\mathbf{p}$ \\
\hline ACA before & Contralateral & 1.33 & 0.35 & 2.551 & 0.011 \\
ACA after & & 1.26 & 0.35 & & \\
MCA before & Contralateral & 2.75 & 0.42 & 2.769 & 0.006 \\
MCA after & & 2.66 & 0.45 & & \\
PCA before & Contralateral & 1.23 & 0.14 & 3.059 & 0.002 \\
PCA after & & 1.15 & 0.15 & & \\
ACA before & Ipsilateral & 1.29 & 0.31 & 3.516 & 0.000 \\
ACA after & & 1.46 & 0.29 & & \\
MCA before & Ipsilateral & 2.77 & 0.50 & 3.621 & 0.000 \\
MCA after & & 2.96 & 0.51 & & \\
PCA before & Ipsilateral & 1.31 & 0.11 & 2.824 & 0.005 \\
PCA after & & 1.24 & 0.14 & & \\
\hline
\end{tabular}

mide challenge between the ICA and vertebrobasilar circulation in patients suffering from chronic CAS; 2) within ICA territory ACAs and MCAs responses to acetazolamide vary in the affected and not affected side.

Posterior carotid arteries diameters decline is most likely the result of blood steal effect due to higher $\mathrm{CO}_{2}$ reactivity in ACAs and MCAs territories. Therefore, our study supports earlier physiological reports suggesting regional differences in $\mathrm{CO}_{2}$ reactivity $[16,17]$. Nevertheless, both Sato et al. [16] and Skow et al. [17] used Doppler ultrasound and measured CBF velocity as a surrogate of CBF in healthy subjects. Doppler ultrasound as a method to estimate CBF is even more questionable in patients with chronic artery stenosis due to turbulent blood flow in the area of stenosis $[7,11,14]$. Therefore, our study, to the best of our knowledge, provides morphometric evaluation of the PCAs reactivity for the first time.

Interestingly, our results with respect to ACA and MCA reactivity are in opposition to most of the available studies, for example $[10,13,20]$. The maximum vasodilatory effect of acetazolamide is usually reached later in the stenosed ICAs than in the contralateral ICAs of patients and the ICAs of healthy control subjects $[10,13]$. In the Hartkamp et al. [10] study in the stenosed ICAs a stepwise decrease in blood flow was observed in the 20- to 25-min and the 25- to 30- $\mathrm{min}$ intervals which may indicate that the vasodilatory 


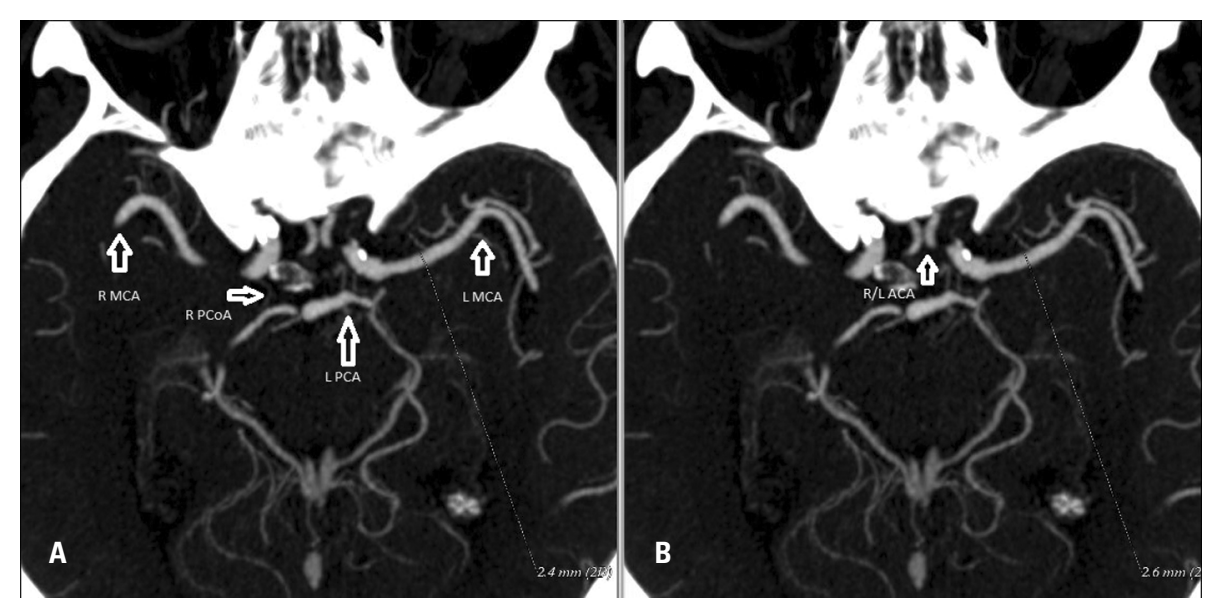

Figure 1. Computed tomography angiography (maximum intensity projection) of Willis arteries in axial plane before (A) and after (B) acetazolamide administration — measurement on the left middle cerebral artery; ACA — anterior cerebral artery; MCA — middle cerebral artery; L PCA — left posterior cerebral artery; R PCoA — right posterior communicating artery; L — left; R — right.

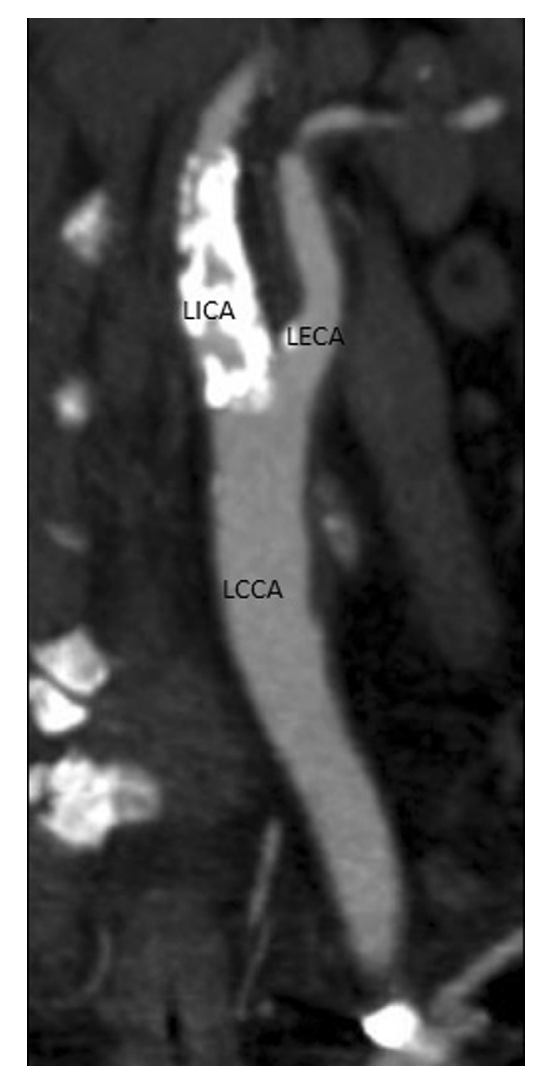

Figure 2. Computed tomography angiography (3-dimensional reconstruction) in coronal plane - the left internal carotid artery (LICA) is narrowed approximately $80 \%$ by atherosclerotic plaques; LCCA — left common carotid artery; LECA — left external carotid artery.

effect of acetazolamide is wearing off. Our study may suggest that the effect of acetazolamide may persist much longer. It should be nevertheless noticed that Hartkamp et al. [10] measured CBF, while we focused on the ACA and MCA diameters.
Alternatively, our population consisted of patients with chronic (over 5 years) CAS $\geq 90 \%$ on the ipsilateral side and, $\leq 50 \%$ on the contralateral side. The Hartkamp et al. [10] population were much healthier with an unilateral symptomatic ICA stenosis $>50 \%$. Finally, we included only patients with fully developed CoW. Most likely, all patients in studied population had well-developed collateral flow as the duration of CAS was longer than 5 years $[3,15,21]$. Therefore our population may be characterised by generally delayed reactivity, while at the same time being relatively well adapted to low CBF. Such reasoning would be in line with results from our previous study on similar population [7]. Taken together, we may observe higher delayed reactivity on the ipsilateral side to stenosis.

Finally, the Bayliss effect cannot be excluded [9]. Intravascular flow can cause vasoconstriction $[4,5]$. This effect shall be distinguished from the myogenic (stretch-related) constriction that follows a rise in intravascular pressure. The phenomenon has been observed only in isolated artery experiments. In vivo, increases in pressure and flow often occur at the same time in the same vascular bed; however, acetazolamide challenge represents such an unique condition when the flow augments while blood pressure remains stable $[1,8]$. Contralateral ACA and MCA may be more prone to such effect.

The study has several limitations. The CTA used to measure the diameters of the intracerebral vessels before and after acetazolamide was not cardiac-gated. Pulsatile flow could, therefore, have caused signal-intensity loss and ghosting artefacts in the phase-encoding direction. We did not measure peripheral blood pressure in this study during the examinations. The acetazolamide chal- 
lenge typically does not evoke changes in blood pressure in humans [1] or in animals [8]. Therefore it is unlikely that such changes occurred in the present study. We did not assess the intracranial artery stenosis. However, such stenosis is relatively rare in European population [12].

Computed tomography remains underutilised, in part, due to concerns raised over high radiation dose. However, a new method to create an ultra-low-dose virtual perfusion computer tomography by enhancing it with CTA information seems to be under way [18]. While magnetic resonance angiography can be used as a screening method, CTA allowing almost isotropic resolution of the images. Consequently morphometric evaluations of cerebral arteries using this technique may become "standard of care" in the near future.

\section{CONCLUSIONS}

In conclusion, there are significant differences in reactivity to acetazolamide challenge between the ICA and vertebrobasilar circulation in patients suffering from chronic CAS. Furthermore, we proved with morphometric assessment that within ICA, territory ACA and MCA responses vary in the affected and not affected side.

\section{REFERENCES}

1. Aamand R, Skewes J, Møller A, et al. Enhancing effects of acetazolamide on neuronal activity correlate with enhanced visual processing ability in humans. Neuropharmacology. 2011; 61(5-6): 900-908, doi: 10.1016/j.neuropharm.2011.06.010, indexed in Pubmed: 21736887.

2. Ainslie PN, Duffin J. Integration of cerebrovascular $\mathrm{CO} 2$ reactivity and chemoreflex control of breathing: mechanisms of regulation, measurement, and interpretation. Am J Physiol Regul Integr Comp Physiol. 2009; 296(5): R1473-R1495, doi: 10.1152/ajpregu.91008.2008, indexed in Pubmed:19211719.

3. Barnett HJ. Hemodynamic cerebral ischemia. An appeal for systematic data gathering prior to a new EC/IC trial. Stroke. 1997; 28(10): 1857-1860, indexed in Pubmed: 9341684.

4. Bevan JA, Joyce EH. Flow-dependent contraction observed in a myograph-mounted resistance artery. Blood Vessels. 1988; 25(5): 261-264, doi:10.1159/000158738, indexed in Pubmed: 3167225.

5. Bevan JA, Joyce EH. Saline infusion into lumen of resistance artery and small vein causes contraction. Am. J. Physiol. 1990; 259(1 Pt 2): H23-H28, indexed in Pubmed: 2375410.

6. Bokkers RPH, Wessels FJ, van der Worp HB, et al. Vasodilatory capacity of the cerebral vasculature in patients with carotid artery stenosis. AJNR Am J Neuroradiol. 2011; 32(6): 1030-1033, doi: 10.3174/ajnr.A2438, indexed in Pubmed: 21393403.

7. Frydrychowski AF, Winklewski PJ, Szarmach A, et al. Nearinfrared transillumination back scattering sounding--new method to assess brain microcirculation in patients with chronic carotid artery stenosis. PLoS ONE. 2013; 8(4): e61936, doi: 10.1371/journal.pone.0061936, indexed in Pubmed: 23613977.

8. Frydrychowski AF, Wszedybyl-Winklewska M, Bandurski T, et al. Flow-induced changes in pial artery compliance registered with a non-invasive method in rabbits. Microvasc. Res. 2011; 82(2): 156-162, doi: 10.1016/j.mvr.2011.05.005, indexed in Pubmed: 21658393.

9. Garcia-Roldan JL, Bevan JA. Augmentation of endothelium-independent flow constriction in pial arteries at high intravascular pressures. Hypertension. 1991; 17(6 Pt 2): 870-874, doi: 10.1161/01. hyp.17.6.870, indexed in Pubmed: 2045168.

10. Hartkamp NS, Hendrikse J, van der Worp HB, et al. Time course of vascular reactivity using repeated phase-contrast MR angiography in patients with carotid artery stenosis. Stroke. 2012; 43(2): 553-556, doi: 10.1161/STROKEAHA.111.637314, indexed in Pubmed: 22052518.

11. Kamouchi M, Kishikawa K, Okada Y, et al. Poststenotic flow and intracranial hemodynamics in patients with carotid stenosis: transoral carotid ultrasonography study. AJNR Am J Neuroradiol. 2005; 26(1): 76-81, doi: 10.1159/000075777, indexed in Pubmed: 15661705.

12. Mattioni A, Cenciarelli S, Biessels G, et al. Prevalence of intracranial large artery stenosis and occlusion in patients with acute ischaemic stroke or TIA. Neurol. Sci. 2014; 35(3): 349-355, doi: 10.1007/s10072-013-1516-4, indexed in Pubmed: 23959530.

13. Murakami M, Yonehara T, Takaki A, et al. Evaluation of delayed appearance of acetazolamide effect in patients with chronic cerebrovascular ischemic disease: feasibility and usefulness of SPECT method using triple injection of ECD. J Nucl Med. 2002; 43(5): 577-583, indexed in Pubmed:11994517.

14. Razavi A, Shirani E, Sadeghi MR. Numerical simulation of blood pulsatile flow in a stenosed carotid artery using different rheological models. J Biomech. 2011; 44(11): 2021-2030, doi: 10.1016/j.jbiomech.2011.04.023, indexed in Pubmed: 21696742.

15. Rothwell PM, Eliasziw M, Gutnikov SA, et al. Carotid Endarterectomy Trialists' Collaboration. Analysis of pooled data from the randomised controlled trials of endarterectomy for symptomatic carotid stenosis. Lancet. 2003; 361(9352): 107-116, doi: 10.1016/s0140-6736(03)12228-3, indexed in Pubmed: 12531577.

16. Sato K, Sadamoto T, Hirasawa Ai, et al. Differential blood flow responses to $\mathrm{CO}$ in human internal and external carotid and vertebral arteries. J. Physiol. (Lond.). 2012; 590(14): 3277-3290, doi: 10.1113/jphysiol.2012.230425, indexed in Pubmed: 22526884

17. Skow RJ, MacKay CM, Tymko MM, et al. Differential cerebrovascular CO reactivity in anterior and posterior cerebral circulations. Respir Physiol Neurobiol. 2013; 189(1): 76-86, doi: 10.1016/j.resp.2013.05.036, indexed in Pubmed: 23774143

18. Tong $E$, Wintermark M. CTA-enhanced perfusion CT: an original method to perform ultra-low-dose CTA-enhanced perfusion CT. Neuroradiology. 2014; 56(11): 955-964, doi: 10.1007/ s00234-014-1416-1, indexed in Pubmed: 25085013.

19. Vorstrup S, Henriksen L, Paulson OB. Effect of acetazolamide on cerebral blood flow and cerebral metabolic rate for oxygen. J. Clin. Invest. 1984; 74(5): 1634-1639, doi: 10.1172/JCI111579, indexed in Pubmed: 6501565.

20. Vorstrup S, Brun B, Lassen NA. Evaluation of the cerebral vasodilatory capacity by the acetazolamide test before EC-IC bypass surgery in patients with occlusion of the internal carotid artery. Stroke. 1986; 17(6): 1291-1298, doi: 10.1161/01. str.17.6.1291, indexed in Pubmed: 3492787.

21. Waaijer A, van Leeuwen MS, van Osch MJP, et al. Changes in cerebral perfusion after revascularization of symptomatic carotid artery stenosis: CT measurement. Radiology. 2007; 245(2): 541-548, doi: 10.1148/radiol.2451061493, indexed in Pubmed: 17848682. 